Artículo

\title{
Análisis proximal de accesiones de calabaza chihua en la Península de Yucatán
}

\author{
José Luis Ruiz García ${ }^{1}$ \\ Antonio Villalobos-González ${ }^{2 \S}$ \\ William Cetzal $\mathrm{Ix}^{1}$ \\ Mónica Beatriz López-Hernandez ${ }^{1}$ \\ María Alma Rangel-Fajardo ${ }^{3}$ \\ José Ángel García-Sandoval ${ }^{4}$
}

${ }^{1}$ Instituto Tecnológico de Chiná. Calle s/n entre 22 y 28, Chiná, Campeche, México. CP. 24520. Tel. 981 8272082, ext. 103. (betymonic@ @otmail.com; jlruiz15@hotmail.com, rolito22@ hotmail.com) 22Campo Experimental Edzná-INIFAP. Carretera Campeche-Pocyaxum km 15.5, Campeche, México. CP. 24520. Tel. 55 38718700, ext. 100 y 310. ${ }^{3}$ Campo Experimental Mococha-INIFAP. Carretera Mérida-Motul km 25, Mococha, Yucatán, México. CP. 97454. Tel. 55 38718700, ext. 88216 y 88213. (rangel.alma@ inifap.gob.mx). ${ }^{4}$ Campo Experimental Chetumal-INIFAP. Carretera Chetumal-Bacalar km 25, Othón Blanco, Quintana Roo, México. CP. 77000. Tel. 55 38718700, ext. 88411. (garcia.angel@inifap.gob.mx).

${ }^{\S}$ Autor para correspondencia: villalobos.antonio@inifap.gob.mx.

\section{Resumen}

El trabajo tuvo como objetivo determinar el contenido de proteína y materia seca en la semilla

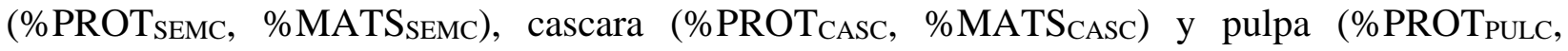
$\%$ MATS $_{\text {PULC }}$ ), y porcentaje de carbohidratos $\left(\% \mathrm{CARB}_{\mathrm{CA}}\right)$, grasa (\%GRAS $\mathrm{CRU}$ ) y ceniza $\left(\% \mathrm{CEN}_{\mathrm{CA}}\right)$ en la semilla de seis accesiones de calabaza chihua (Cucurbita argyrosperma Huber), nativas de la Península de Yucatán. Las accesiones evaluadas fueron: Becal, Edzná, Pixoyal, Chihua local, CACAO y CECHET. La siembra fue realizada en junio de 2017 en Pocyaxum, San Francisco de Campeche, México, con diseño de bloques al azar con tres repeticiones, se realizó análisis de varianza y comparaciones de medias con prueba de Tukey $(p \leq 0.05)$. La unidad experimental consistió en tres surcos de $6 \mathrm{~m}$ de longitud, separados a $2.4 \mathrm{~m}$ y $1.25 \mathrm{~m}$ entre planta. Para la determinación de proteínas se empleó el método Kjeldahl, y siguiendo el proceso descrito en AOAC (2000). La accesión CECHET mostro mayor $(p \leq 0.05) \%$ PROTESEMC, \%PROT ${ }_{\text {CASC, }}$ \%MATS SEMC y $\%$ MATS $_{\text {PULC }}$ con 33.6, 14.4, 64.9 y 4.29\%, respectivamente. Edzná, Becal, Local Yucatán presentaron 3.8\% PROT PULC menos $(p \leq 0.05)$ en comparación a las accesiones CECHET, CACAO y Pixoyal. Edzná presento mayor $(p \leq 0.05) \%$ GRAS $_{\mathrm{CRU}}$ con $45.3 \%$. Becal, presento mayor $(p \leq 0.05) \% \mathrm{CARB}_{\mathrm{CA}}$ con $34.7 \%$. El \%MATS ${ }_{\text {CASC }}$ de la accesión CACAO (12.5\% MATS $\left._{\text {CASC }}\right)$ superan $(p \leq 0.05)$ al resto de las accesiones. No hubo diferencias $(p \leq 0.05)$ para $\% \mathrm{CEN}_{\mathrm{CA}}$. La accesión que sobresalió fue CECHET, con mayor porcentaje de proteína en semilla, cascara, pulpa y materia seca en semilla y pulpa. Se identificaron accesiones con aporte nutricional para consumo que pueden ser alternativa para elaboración de distintos alimentos.

Palabras clave: Cucurbita argyrosperma Huber, biodiversidad, calabaza nativa.

Recibido: septiembre de 2020

Aceptado: noviembre de 2020 


\section{Introducción}

Las especies cultivadas del género Cucurbita (Cucurbitaceae) en México y el resto de Mesoamérica son las plantas que, de forma general, se denominan calabazas y chilacayotes (Mera et al., 2011). Cucurbita (Cucurbitaceae) es un género relativamente reciente, que surgió en Norte América hace 16 millones de años y sus especies cultivadas mantienen una alta variación genética; Cucurbita pepo es la especie que presenta mayor variación genética, variación asociada a dos domesticaciones independientes, una en el norte de México, y otra en el Sureste de los Estados Unidos de América (Eguiarte et al., 2018). En otra especie, Cucurbita argyrosperma, sus poblaciones de la Península de Yucatán representan un pool genético diferenciado del resto de la especie (Eguiarte et al., 2018).

Los estudios realizados en las especies de calabaza, especialmente investigaciones direccionados a características físicas y químicas del fruto ha recibido considerable atención para diversificar los productos finales (Rössel et al., 2018). De estos productos se puede aprovechar la semilla debido a que son ricas en compuestos beneficiosos para la salud como polisacáridos, carotenos, sales minerales, vitaminas y otros (Fu et al., 2007). Vaštag et al. (2014) indica que la fracción mayor de estas proteínas está representada por cucurbitinas. Las proteínas de semilla de calabaza son ingredientes deseables en los productos alimenticios, al presentar efectos antimicrobianos, anticarcinógenos y al aliviar los efectos perjudiciales asociados con la desnutrición proteínica (Bučko et al., 2016).

El valor nutricional de las semillas de calabaza se basa en un alto contenido proteínico (25-51\%) y un alto porcentaje de aceite (40 y $60 \%$ ), el $46.9 \%$ del aceite proviene de los ácidos grasos oleico, $40.5 \%$ linolénico, $17.4 \%$ de palmiótico y esteárico y 0.60 a $0.75 \mathrm{~g}$ de monoinsaturados y ácidos polisaturados (Rezig et al., 2016). Consecuentemente, las proteínas vegetales se utilizan cada vez más como fuentes no convencionales de proteínas para desempeñar funciones en formulaciones de alimentos (Abdel, 2006).

Los ingredientes basados en proteínas de la calabaza aún no se han introducido a una escala significativa en las aplicaciones alimentarias y aunque existen un gran flujo de datos sobre las propiedades funcionales de diversas proteínas de especies de calabaza, actualmente son pocos los datos disponibles, esto se debe probablemente a la información limitada sobre las propiedades estructurales y funcionales de las proteínas de calabaza (Rezig et al., 2016).

Particularmente en la Península de Yucatán, la información sobre calabaza y en especial de especies nativas de calabaza chihua y su contenido de proteína es insuficiente. Por lo tanto, el objetivo del presente trabajo fue determinar el contenido de proteína y materia seca en la semilla, cascara y pulpa y porcentaje de carbohidratos, grasa y ceniza en la semilla de seis accesiones de calabaza chihua (Cucurbita argyrosperma Huber), nativas de la Península de Yucatán

\section{Materiales y métodos}

El experimento en condiciones de campo se realizó en una superficie de terrenos aledaños a la comunidad de Pocyaxum, Municipio de San Francisco de Campeche, Campeche, México, cuyas coordenadas geográficas son: 19.7333 norte, -90.3583 oeste y a una altitud de $30 \mathrm{msnm}$. En el ciclo 
de primavera-verano 2017 bajo condiciones de temporal, sobre suelos rojos-arcillosos-profundos, denominados Kancab o K'aancab en la clasificación de suelos elaborados por los antiguos Mayas y llamados Luvisoles en la clasificación de la FAO-UNESCO (1970).

El estudio se evaluaron seis accesiones de calabaza chihua (Cucurbita argyrosperma Huber) utilizadas a nivel regional, tres de ellas pertenecen al estado de Campeche denominadas: Becal, Edzná y Pixoyal, de Yucatán se incluyó a la accesión Chihua local y de Quintana Roo se agregó a CACAO y CECHET.

Se sembraron los diferentes cultivares en junio 2017. Se utilizó un diseño experimental de bloques completos al azar con tres repeticiones. La unidad experimental consistió en tres surcos de seis metros de longitud, separados a $2.4 \mathrm{~m}$ uno del otro y $1.25 \mathrm{~m}$ entre planta, al depositar dos plantas por mata en suelo húmedo a $3 \mathrm{~cm}$ de profundidad y posteriormente a los tres días de la emergencia se clareo a una planta, respectivamente.

Se fertilizó con la fórmula 27N-69P-60K al aplicar de forma manual a los 15 días después de la siembra (dds) con $150 \mathrm{~kg}$ de fosfato diamónico (18-46-00) en mezcla con $100 \mathrm{~kg}$ de cloruro de potasio. Antes de la emergencia se realizó la aplicación de glifosato en dosis de $2 \mathrm{~L} \mathrm{ha}^{-1}$.

En relación con la fase de laboratorio, todos los frutos de la parcela experimental se cosecharon a los 93 dds en etapa de madurez fisiológica y se trasportaron al laboratorio del Instituto Tecnológico de China, Campeche, México. Seguidamente se seleccionaron de forma aleatoria cinco frutos de cada una de las accesiones evaluadas. Posteriormente los frutos fueron diseccionados en pulpa de la cascara, también se obtuvieron las semillas, pesándose $100 \mathrm{~g}$ de cascara, pulpa y semillas en una balanza digital Highland ${ }^{\circledR}$ y posteriormente se colocaron en una estufa de convección natural Memmert ${ }^{\circledR}$ para su deshidratación, se colocaron en charolas de aluminio previamente identificadas durante 96 horas a una temperatura de $65^{\circ} \mathrm{C}$.

Una vez deshidratados se colocaron en un desecador hasta que se enfriaron, se pesaron y se molieron en un molino Pulvex ${ }^{\circledR}$. Las muestras ya en polvo, se colocaron en bolsas transparentes de plástico y se conservaron a temperatura ambiente hasta el momento de los análisis. En este contexto, el trabajo integró la evaluación del contenido de proteína de las accesiones de calabaza chihua en la semilla, cascara y pulpa. Para la determinación de proteínas se empleó el método Kjeldahl, utilizando el equipo Kjeldahl y siguiendo el proceso descrito en AOAC (2000).

Este método consiste en tres pasos: a) digestión de la muestra; b) destilación del nitrógeno (N); y c) titulación, que a continuación se describen: a) digestión: se pesó 1 g de selenio y 1 g de muestra y se colocaron dentro de los tubos de ensayo del sistema compacto de digestión (Raypa MBC-12 TB 26300 700), posteriormente se añadieron $10 \mathrm{ml} \mathrm{de} \mathrm{H}_{2} \mathrm{SO}_{4}$ a los mismos tubos.

La técnica se estandarizó utilizando un tiempo de digestión total de 3 horas y 15 min divididos de acuerdo con las siguientes actividades: 15 min para llegar a $150{ }^{\circ} \mathrm{C}$, que se mantuvo durante 30 min; 15 min para llegar a $270^{\circ} \mathrm{C}$, que se mantuvo durante $30 \mathrm{~min}, 15 \mathrm{~min}$ para incrementar a $370^{\circ}$, que se mantuvo durante 90 min (AOAC, 2000). 
Al finalizar se dejó enfriar durante 20 a 30 min para comenzar el proceso de destilación. b) destilación: al término de la digestión, se agregó al tubo de destilación la muestra, también $25 \mathrm{ml}$ de $\mathrm{NaOH}$ y $75 \mathrm{ml}$ de $\mathrm{H}_{2} \mathrm{O}$ destilada.

No obstante, el equipo (destilador de N proteína tipo flash, marca Raypa DNP-1500-MP) agrega $25 \mathrm{ml}$ más de $\mathrm{H}_{2} \mathrm{O}$ destilada durante el proceso. Previamente, se colocó en un matraz de $250 \mathrm{ml}$, $25 \mathrm{ml} \mathrm{de} \mathrm{H}_{3} \mathrm{BO}_{3}$ al $4 \%$ y 3 gotas del indicador Tashiro.

Posteriormente se configuró el proceso con tiempo de espera de $30 \mathrm{~s}$, vapor al $100 \%$ y 8 min de destilado para recolectar $250 \mathrm{ml}$ de muestra. c) titulación: el proceso se realizó con $\mathrm{HCl}$ al $0.25 \%$ $\mathrm{N}$, la muestra obtenida de la destilación se sometió a titulación hasta que haga el primer cambio de color (ligeramente rosa). El cálculo de $\mathrm{N}$ obtenido se realizó utilizando la siguiente fórmula.

$$
\% \mathrm{~N}=\frac{(\text { Gasto de la muestra-gasto del blanco) (normalidad del ácido) (1.4) x } 100}{\text { Peso de la muestra }(\mathrm{g})}
$$

El \% de proteína se calculó con relación al \% de $\mathrm{N}$ con la siguiente ecuación: \%proteína $=(\% \mathrm{~N})$ (6.25). Donde: $\% \mathrm{~N}=$ porcentaje de $\mathrm{N} ; 6.25=$ constante para hortalizas (AOAC, 2000). Cada variable se sometió a un análisis varianza con el programa SAS para Windows Versión 9.0 (SAS, 2002) en forma individual.

La comparación de medias se realizó con la prueba de Tukey $(p \leq 0.05)$. Se utilizó la prueba de Shapiro-Wilk y t-student para los supuestos de normalidad de datos y homogeneidad de varianza.

\section{Resultados y discusión}

\section{Porcentaje de proteína en la semilla de calabaza (\%PROT}

El contenido de proteína en las semillas de las accesiones de calabaza chihua es estadísticamente diferentes $(p \leq 0.05)$. La accesión que mostró mayor $(p \leq 0.05) \%$ PROT $_{\text {SEMC }}$ fue CECHET con $33.6 \%$ en comparación con Becal, Pixoyal, CACAO, Edzná y Local Yucatán, que obtuvieron en ese orden 26.9, 29.6, 30.3, 30.5 y 32.7\% PROT SEMC, respectivamente (Figura 1).

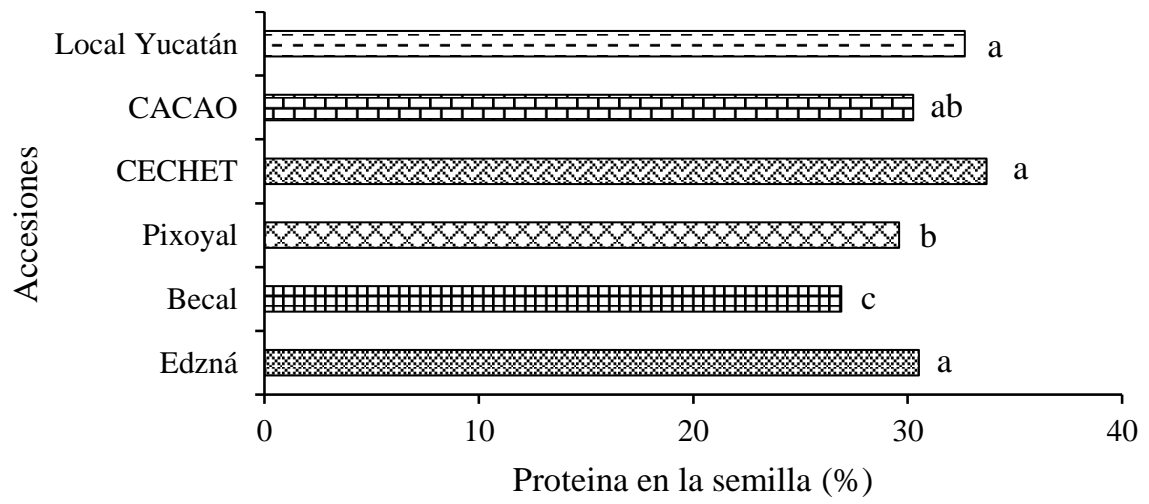

Figura 1. Porcentaje de proteína en la semilla de calabaza nativa. Medias con la misma letra(s) entre tratamientos son estadísticamente iguales con un $\alpha=0.05$. 
La variabilidad en el contenido de PROT $_{\text {SEMC }}$ se debe a la funcionalidad de las proteínas de origen vegetal que dependen de las características químicas inherentes a la semilla (Mattil, 1973). Las propiedades funcionales son resultado de las propiedades fisicoquímicas de las proteínas, que influyen de modo específico sobre su comportamiento (Hernández et al., 2014).

\section{Porcentaje de proteína en la cascara de calabaza (\%PROTCASc)}

Los datos del estudio muestran que la accesión CECHET procedente del estado de Quintana Roo presentó el mayor $(p \leq 0.05) \%$ PROT $_{\text {CASC }}$ con $14.4 \%$. Applequist et al. (2006) realizaron un estudio en accesiones de calabaza en campo y observaron que una accesión del estado de Campeche

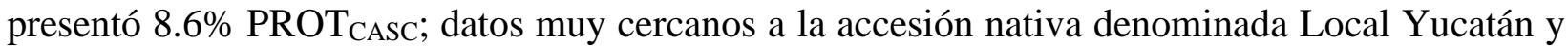
Becal, que presentaron un \%PROT ${ }_{\text {CASC }}$ de 9.3. y 9.4\%, respectivamente (Figura 2). Young et al. (2012) señala que el \%PROT ${ }_{\text {CASC }}$ de calabaza depende de la especie cultivada en la región y condiciones que son cultivadas, ya que se ha observado que la cascara de Cucurbita maxima contiene más proteína que Cucurbita pepo o Cucurbita mochata.

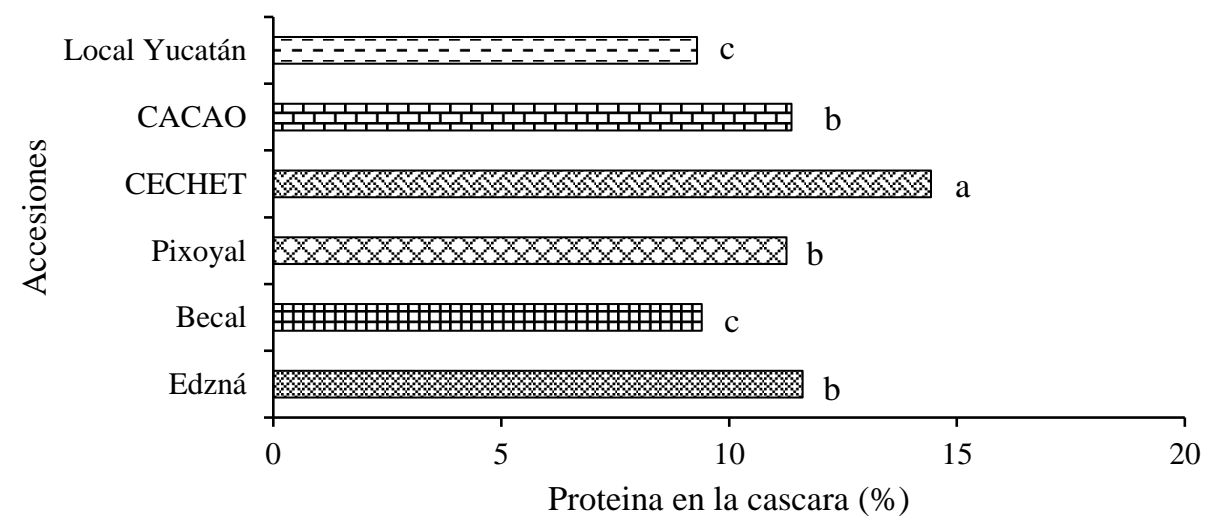

Figura 2. Porcentaje de proteína en la cascara de calabaza nativa. Medias con la misma letra(s) entre tratamientos son estadísticamente iguales con un $\alpha=0.05$.

Estudios realizados por Ziaul et al. (2019) revelan que existen cultivares nativos de calabaza Cucurbita maxima que presentan mayor contenido de proteína en la cascara en comparación con variedades hibridas y estas diferencias son atribuidas a las diferencias entre las especies y variedades Cucurbita spp., cultivado en diferentes áreas del mundo.

\section{Porcentaje de proteína en la pulpa de calabaza (\%PROTPuLC)}

Estudios realizados por Kim et al. (2012) en campo, observaron que, un material de calabaza $(C$. pepo) presento en promedio $1 \%$ PROT $\mathrm{PULC}$. Mientras que en el presente trabajo se obtuvo una variación $(p \leq 0.05)$ en la cantidad del \%PROT PULC; al observa que Edzná, Becal, Local Yucatán presentaron 3.8\% PROT PULC menos que las accesiones CECHET, Cacao y Pixoyal (Figura 3).

La variación que presentaron la accesión CECHET, Cacao y Pixoyal en el \%PROT PULC en comparación con el resto puede atribuirse a las diferencias en los componentes químicos que persisten en cada una de ellas, que a su vez está relacionada con la región donde se cultiva (El y Mulhieddine, 2019). 


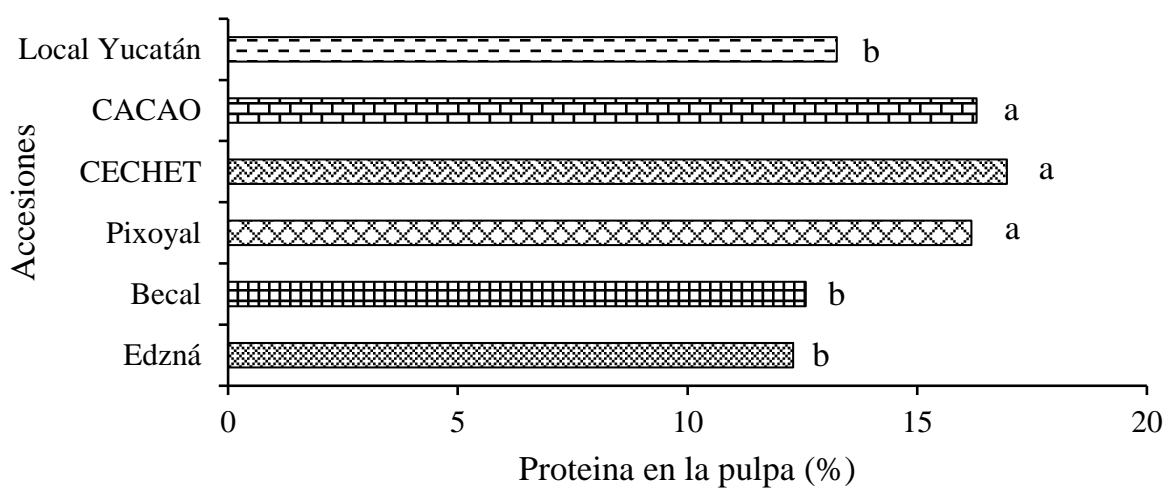

Figura 3. Porcentaje de proteína en la pulpa de calabaza nativa. Medias con la misma letra(s) entre tratamientos son estadísticamente iguales con un $\alpha=0.05$.

\section{Porcentaje de grasa cruda en la semilla de calabaza (\%GRAScru)}

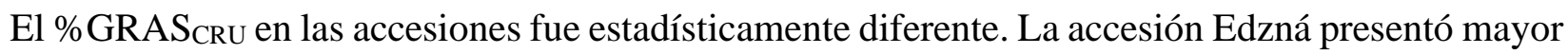
$(p \leq 0.05)$ cantidad de $\%$ GRAS $_{\text {CRU }}$, que CACAO, Local Yucatán, Pixoyal, Becal y CECHET con

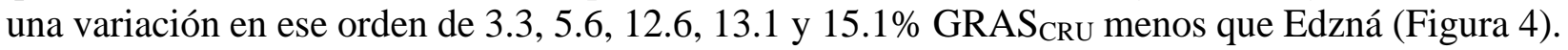

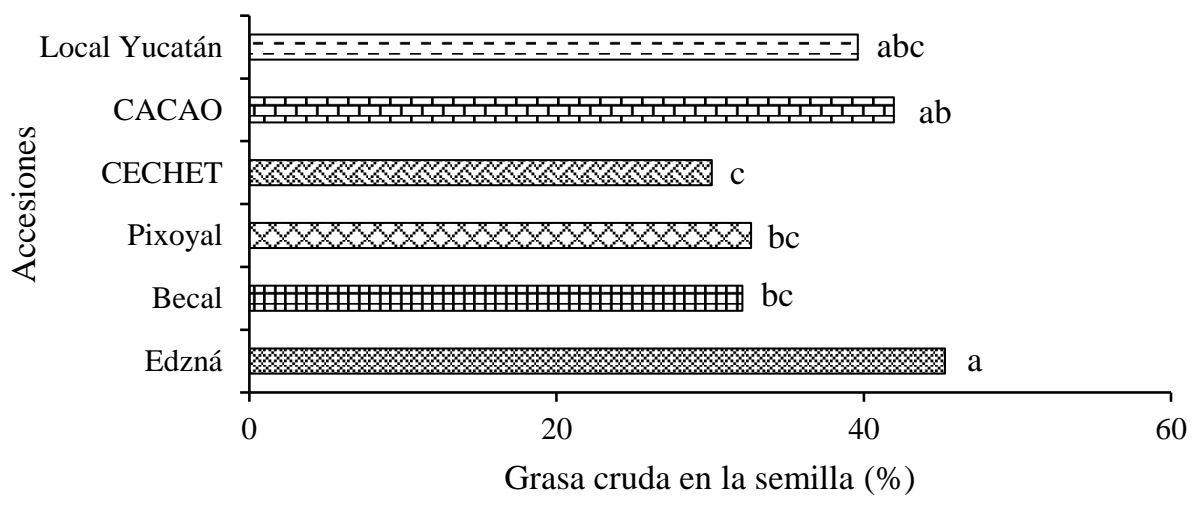

Figura 4. Porcentaje de grasa cruda de las accesiones nativas de calabaza. Medias con la misma letra(s) entre tratamientos son estadísticamente iguales con un $\alpha=0.05$.

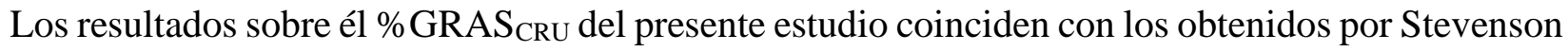
et al. (2007), que observaron valores entre 36 a $40.1 \%$ GRAS $_{\mathrm{CRU}}$ en cultivares de calabaza (Cucurbita maxima D). Mientras que en los estudios realizados por Jarret et al. (2013) se obtuvo un $13.5 \%$ (24.4 a $32.9 \%$ ) más de grasa cruda en la semilla que los obtenidos en el presente trabajo.

Entre los aspectos que influyen sobre la variabilidad en el \%GRAS $\mathrm{GRU}_{\mathrm{C} U}$ se señala que la semilla contiene diferentes cantidades de minerales como $\mathrm{Fe}, \mathrm{Ca}, \mathrm{Mg}, \mathrm{Zn}, \mathrm{P}, \mathrm{K}$ y $\mathrm{Cu}$, al igual que anti nutrientes como oxalatos, cianuros, tianinos y filatos, que propician una variación en el \% GRAS $\mathrm{CRU}_{\mathrm{C}}$ de la calabaza buido (Telfairia occidentalis) (Ekpedeme et al., 1999).

Otro factor que pudo incidir en la variación del \%GRSACRU es la procedencia de las accesiones, debido a que las características físicas de grasa y aceite son dependientes de factores tales como la semilla o planta de procedencia, grado de insaturación, tamaño de las cadenas de carbono, formas isoméricas de los ácidos grasos y estructura molecular de los triglicéridos (Lawson, 1999). 


\section{Porcentaje de ceniza en la semilla de calabaza (\% CENCa)}

En relación con el $\% \mathrm{CEN}_{\mathrm{CA}}$, no se presentaron diferencias significativas $p \leq 0.05$ entre las accesiones (Figura 5). Los valores más altos de cenizas obtenidos en esta investigación sobrepasan en $1.8 \%$ a lo obtenido por Gohari et al. (2011) en calabaza (Cucurbita pepo), obteniendo 5.3\%

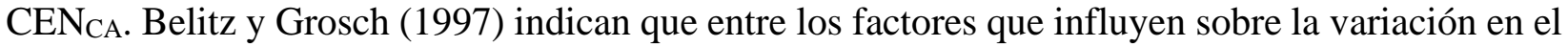
$\% \mathrm{CEN}_{\mathrm{CA}}$ es el contenido de minerales $(\mathrm{P}, \mathrm{Zn}, \mathrm{Cu}$ y $\mathrm{Mg}$ ) que es dado por factores genéticos, climáticos, prácticas de cultivo, composición de suelo y periodo de maduración del fruto a cosecha.

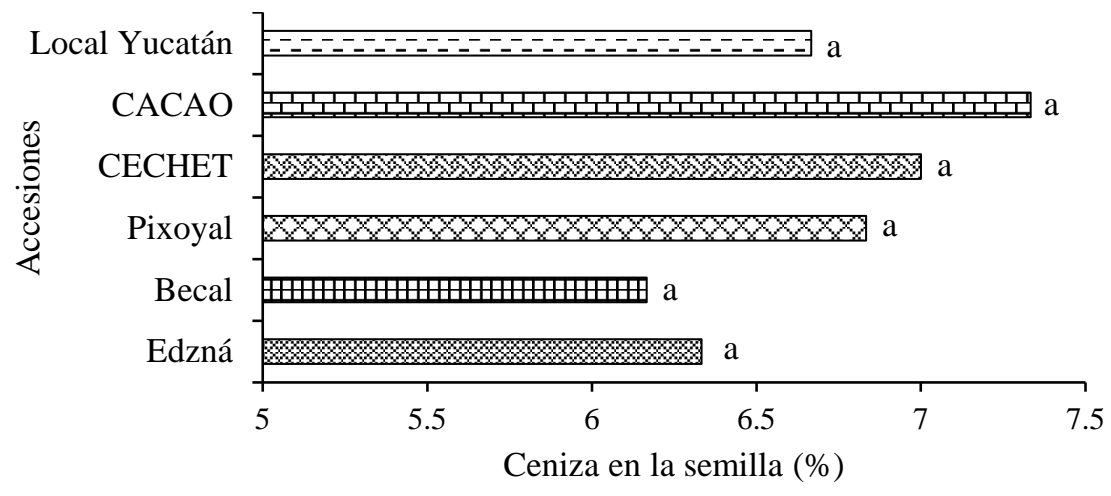

Figura 5. Porcentaje de ceniza en la semilla de las accesiones nativas de calabaza. Medias con la misma letra(s) entre tratamientos son estadísticamente iguales con un $\alpha=0.05$.

\section{Porcentaje de carbohidratos en la semilla de calabaza (\% CARBSE)}

El \%CARB $\mathrm{CE}_{\mathrm{SE}}$ de la accesión Becal es superior $(p \leq 0.05)$ al resto de las accesiones evaluadas. Simultáneamente en el presente estudio, se observó, que Becal obtuvo una variación superior ( $p \leq$

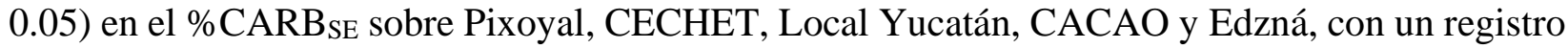
en ese orden de las accesiones de 3.2, 5.6, 11.8, 14.4 y 22.1\% CARB $_{\mathrm{CA}}$ (Figura 6).

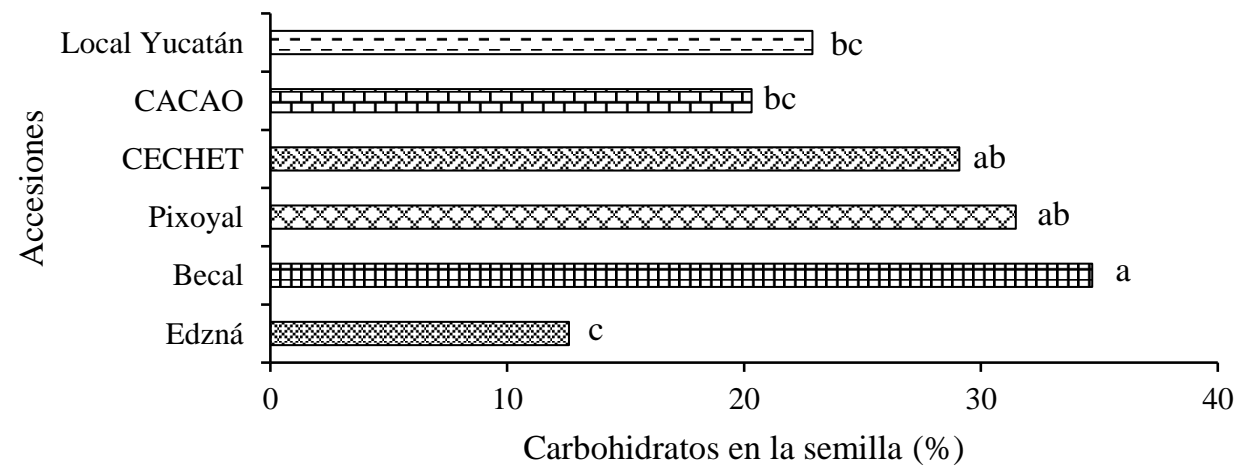

Figura 6. Porcentaje de carbohidratos en las accesiones nativas de calabaza. Medias con la misma letra(s) entre tratamientos son estadísticamente iguales con un $\alpha=0.05$.

Es posible que el \%CARBSE de las accesiones estuvo relacionado a la procedencia y manejo posteriores del cultivo en Quintana Roo, Yucatán y Campeche, semejante a los observados en 13 cultivares de Cucurbita pertenecientes a diversas regiones agroecologías de la zona oriente y centro

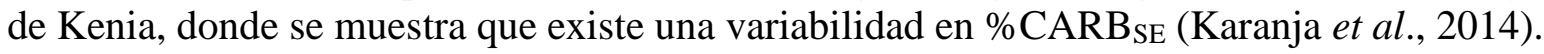


Cerón et al. (2010) indica que, de acuerdo con la diversidad genética, la calidad y cantidad nutricional de la semilla de calabaza es heterogenia y esto se constata con los resultados obtenidos en los estudios realizados por Terazawe et al. (2001) al observar que la calabaza Cucurbita maxima Duch y Cucurbita moschata Duch a medida que sus frutos crecen difieren en el contenido de carbohidratos (azúcares) y este contenido total de azúcar de Cucurbita maxima Duch. aumentó gradualmente después de 30 días desde la floración, mientras que Cucurbita moschata Duch. incrementó de manera constante durante el crecimiento de la fruta.

\section{Porcentaje de materia seca en la semilla de calabaza (\%MATSEMc)}

El contenido de materia seca en la semilla de los cultivares nativos de calabaza de la región es variable, esto permite que algunas especies de calabaza nativas prevalezcan en la región. Por ejemplo, las semillas ortodoxas en algunos casos reducen sus contenidos de humedad a valores menores de 10 y $15 \%$, lo que les permite sobrevivir ante los cambios del ambiente y ser almacenadas por periodos largos (Kermode, 1997).

En este sentido, en el presente trabajo se observó un valor promedio de 55\% de MATS $\mathrm{SEMC}_{\text {entre }}$

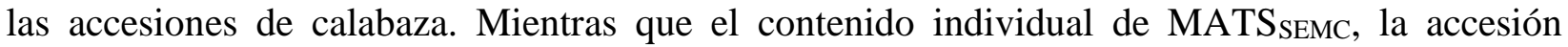
denominada CECHET supero $(p \leq 0.05)$ a Edzná, Local Yucatán, Becal, CACAO y Pixoyal con

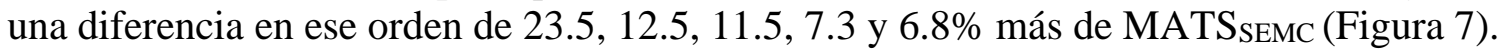

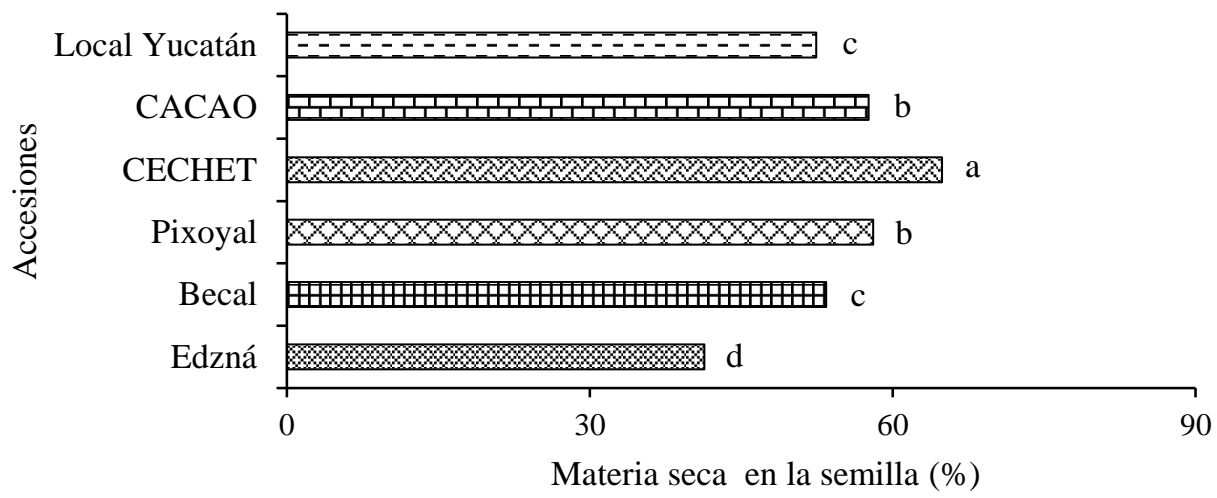

Figura 7. Porcentaje de materia seca en la semilla de calabaza nativa. Medias con la misma letra(s) entre tratamientos son estadísticamente iguales con un $\alpha=0.05$.

\section{Porcentaje de materia seca en la pulpa de calabaza (\%MATSPUL)}

En el presente trabajo, el \%MATS PuLC fluctuaron entre 1.9 a 4.3\%. En este contexto, la accesión CECHET presentó mayor $(p \leq 0.05) \%$ MATS PULC que Edzná, Becal, Pixoyal, Local Yucatán y CACAO con una diferencia en ese orden de 2.4, 2.3, 2, 1.7 y 0.8\% MATS PULC (Figura 8).

Sobre las características nutricionales de la calabaza, excepto en azúcares y acidez. El presente estudio revela que en la Península de Yucatán hay accesiones nativas que presentan una mayor cantidad de materia seca en la pulpa en comparación con otras cultivadas en la región y esta variación es atribuida a que en algunas de las accesiones las características nutricionales de la calabaza se ven afectadas negativamente por el contenido de humedad del fruto que esta alrededor de $90 \%$ (Ortiz et al., 2008), convirtiéndose en un fruto perecedero, de corta vida, con los nutrientes 
diluidos, donde la longevidad del fruto y su capacidad de ser almacenado durante periodos prolongados depende del porcentaje de materia seca (Lacuzzo y Dalla, 2009). Por tanto, la identificación de genotipos con niveles altos de materia seca es fundamental para fortalecer el mejoramiento genético con perspectiva agroindustrial (Ortiz et al., 2013).

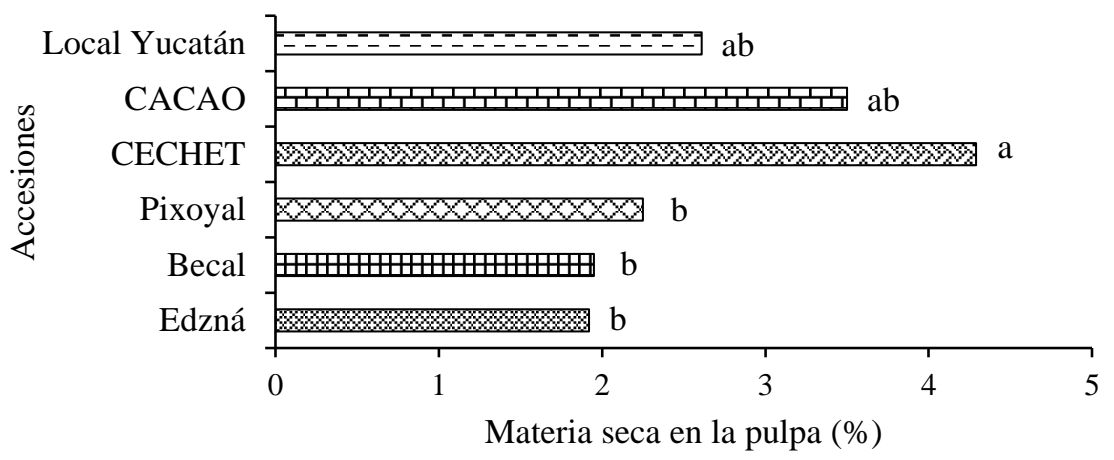

Figura 8. Porcentaje de materia seca en la pulpa de calabaza nativas. Medias con la misma letra(s) entre tratamientos son estadísticamente iguales con un $\alpha=0.05$.

\section{Porcentaje de materia seca en la cascara de calabaza (\% MATScasc)}

Los valores observados en el porcentaje de materia seca en la cascara de la accesión denominada CACAO superan $(p \leq 0.05)$ a los observados en la accesión Pixoyal, Becal, Edzná, Local Yucatán y CECHET con una variación en ese orden de 6.8, 5.5, 5.4, 4.9 y 4.4\% MATS $_{\text {CASC (Figura 9). }}$

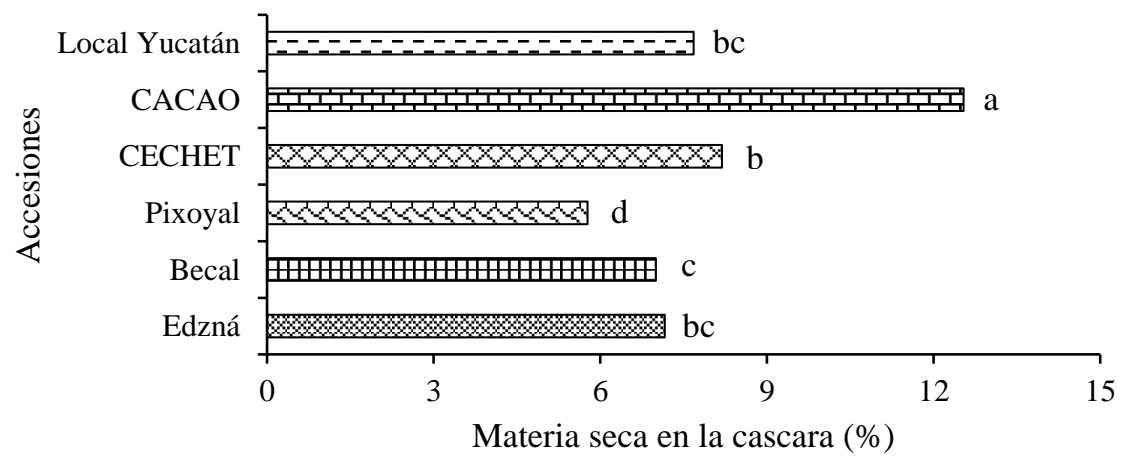

Figura 9. Porcentaje de material seca en la cascara de calabaza nativa. Medias con la misma letra(s) entre tratamientos son estadísticamente iguales con un $\alpha=0.05$.

Estos resultados contrastan con los obtenidos por Lorenzo et al. (2019) al obtener 13.71\% de MATS $_{\text {CASC }}$ con pulpa de calabaza. Dorantes et al. (2016) en accesiones de calabaza nativa (Cucurbita argyrosperma Huber) del estado de Campeche observaron valores de $6.45 \%$ de

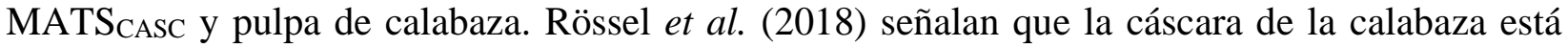

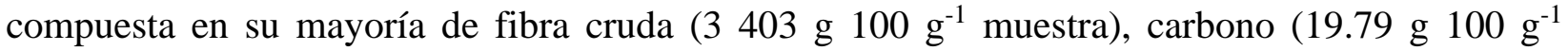
muestra) y calcio (30.057 mg $100 \mathrm{~g}^{-1}$ muestra). 


\section{Conclusiones}

El contenido nutricional de las accesiones de calabaza chihua (Cucurbita argyrosperma Huber) cultivadas en la Península de Yucatán es variable. La accesión que presento mayor contenido nutricional en las partes del fruto fue CECHET de Quintana Roo, al obtener mayor porcentaje de proteína en la semilla, cascara, pulpa y porcentaje de materia seca en la semilla y pulpa. Edzná de Campeche destacó en el contenido de grasa cruda en la semilla. Becal de Campeche fue superior a todas las accesiones en el contenido de carbohidratos.

La accesión CACAO de Quintana Roo presentó mejores resultados para el contenido de materia seca en la cascara. Se identificaron accesiones con aporte nutricional para su consumo que a su vez pueden resultar como alternativa para diversificar su uso en la elaboración de distintos alimentos.

\section{Literatura citada}

Abdel, R. M. K. 2006. Effect of pumpkin seed (Cucurbita pepo L.) Diets on benign prostatic hyperplasia (BPH): chemical and morphometric evaluation in rats. World J. Chem. $1(1): 33-40$.

AOAC. 2000. International. Official Methods of Analysis of AOAC International, 17 ${ }^{\text {th }}$ (Ed.). Arlington USA. 673 p.

Applequist, W. L.; Avula, B.; Schaneberg, B. T.; Wang, Y. H. and Khan, I. A. 2006. Comparative fatty acid content of seeds of four Cucurbita species grown in a common (shared) garden. J. Food Compos. Anal. 19(6-7):606-611.

Belitz, H. D. and Grosch, W. 1997. Química de los alimentos. 2a (Ed.). Zaragoza: Acribia. 1087 p.

Bučko, S. D.; Katona, J. M.; Popović, I. M.; Vaštag, Ž. G. and Petrović, I. B. 2016. Functional properties of pumpkin (Cucurbita pepo) seed protein isolate and hydrolysate. J. Serb. Chem. Soc. 81(1):35-46.

Cerón, G. L.; Legaria, S. P. J.; Villanueva, V. C. y Sahagún, C. J. 2010. Diversidad genética en cuatro especies mexicanas de calabaza (Cucurbita spp.). Rev. Fitotec. Mex. Vol. 33(3):189-196.

Dorantes, J. J.; Flota, B. C; Candelaria, M. B.; Ramírez, M. M. y Crosby, G. M. M. 2016. Calabaza chihua (Cucurbita argyrosperma Huber), alternativa para alimentación animal en el trópico. Agroproductividad. 9(9):33-37.

Eguiarte, L. E.; Rosales, H. H. S.; Redondo, B. J.; Morales, C. G.; Torres, P. L. M.; Sánchez-de la Vega G.; Mondragón, R. K. Y.; Lobo, V. A.; Hernández, M. S.; Planter, A. E.; Sauza, V. y Lira, R. 2018. Domesticación, diversidad y recursos genéticos y genómicos de México: el caso de las calabazas. Tip Revista especializada en ciencias químicobiológicas. 21(2):85-101.

Ekpedeme, U. A.; Bassey, A. N.; Ekaete, U. and Etuk. 1999. Minerals and antinutrients in fluted pumpkin (Telfairia occidentalis Hook f.). Food Chemistry 702000 235-240. Analytical, Nutritional and Clinical Methods Section. Nigeria.

El, K. S. B. and Multhieddine, M. 2019. Nutritional profile and medicinal properties of pumpkin fruit pulp. Chapter. Ed. 1-19 pp.

FAO-UNESCO. 1970. Food and Agricultural Organization of the United Nations. Clave de unidades de suelos Del mundo. Secretaría de Recursos Hidráulicos (SRH). Dirección de Agrología. México, DF. 
Fu, C. L.; Tian, H. J.; Cai, T. Y.; Liu, Y. and Li, Q. H. 2007. Some properties of an acidic proteinbound polysaccharide from the fruit of pumpkin. Foo d Chem. 100(3):944-947.

Gohari, A. M.; Mehrabi, R.; De-Wit, P. J. G. M. and Kema, G. H. J. 2011. MgWor1 is involved in conidia formation, hyphal morphogenesis and pathogenicity of the fungal wheat pathogen Mycosphaerella graminícola. Book of Abstracts In: $8^{\text {th }}$ International Symposium on Mycosphaerella and Stagonospora Diseases of Cereals. Mexico City September. 10-14. $32 \mathrm{p}$.

Hernández, S. B.; Gordillo, M. J. A.; Martínez, S. E. C. y Castro, R. J. 2014. Potencial nutricional de la semilla de calabaza (Cucurbita pepo). In: los alimentos en México y su relación con la salud. Aguilera, O. M.; Ramírez, J. A.; Reynoso, C. R. y Gómez, A. C. (Eds.). $1^{\text {a }}$ Edición. México. 191-211 p.

Jarret, R.; Levy, I.; Potter, T.; Cermak, S. and Merrick, L. 2013. Seed oil content and fatty acid composition in a genebank collection of Cucurbita moschata Duchesne and $C$. argyrosperma C. Huber. Plant. Genet. Resour. 11(2):49-157.

Karanja, K. J.; Mugendi, J. B.; Khamis, M. F. and Muchugi, N. A. 2014. Nutritional evaluation of some Kenyan Pumpkins (Cucurbita spp.). Inter. J. Agric. Forest. 4(3):195-200.

Kermode, A. R. 1997. Approaches to elucidate the basis of desiccation-tolerance in seeds. Seed Sci. Res. 7(2):75-95.

Kim, M. Y.; Kim, E. J.; Kim, Y.; Choi, H. and Lee, B. 2012. Comparison of the chemical compositions and nutritive values of various pumpkin (Cucurbitaceae) species and parts. Nutr. Res. Pract. 6(1):21-27.

Lacuzzo, F. and Dalla, C. L. 2009. Yield performance, quality characteristics and fruit storability of winter squash cultivars in sub-humid areas. Sci. Hortic. 120(3):330-335.

Lawson, H. 1999. Aceites y grasas alimentarios: tecnología, utilización y nutrición. Acribia. Ed. $346 \mathrm{p}$.

Lorenzo, H. R.; Torres, S. N.; Sánchez, S. P.; Herrera, P. J.; Mayrén, M. J. F.; Salinas, R. T.; Rafael, R. G. A. y Maldonado, P. A. M. 2019. Evaluación de las características de calidad y bromatológicas de ensilados elaborados con residuos de calabaza (Cucurbita argyrosperma). Rev. Int. Contam. Ambie. 35(4):957-963.

Mattil, K. F. 1973. Considerations for choosing the right plant proteins. Food product development, 7(6):40-44.

Mera, O. L. M.; Bye, B. R. A.; Villanueva, V. C. y Luna, M. A. 2011. Documento de diagnóstico de las especies cultivadas de Cucurbita L. SAGARPA, SINAREFI, SNICS. México. 79 p.

Ortiz, G. S.; Sánchez, L. J.; Valdés, R. M. P.; Baena, G. D. y Vallejo, C. F. A. 2008. Retención de caroteno total en fruto de zapallo Cucurbita moschata Duch acondicionado por osmodeshidratación y secado. Acta Agron. 57(4):269-274.

Ortiz, G. S.; Vallejo, C. F. A.; Baena, G. D.; Estrada, S. E. I. y Valdés, R. M. P. 2013. Zapallo para consumo en fresco y fines agroindustriales: investigación y desarrollo. Universidad Nacional de Colombia, Sede Palmira. ed Santiago de Cali, Feriva. Colombia. 299 p.

Rezig, L.; Riaublanc, A.; Chouaibi, M.; Guéguen, J. and Hamdi, S. 2016. Functional properties of protein fractions obtained from pumpkin (Cucurbita maxima) Seed. Int. J. Food Prop. 19(1):172-186.

Rössel, D. K.; Ortiz, H. L; Amante, O. A.; Durán, G. H. M. and López, L. A. M. 2018. Physical and chemical characteristics of pumpkin seeds for mechanization and processing. Nova Scientia. 10 (2):61-77.

SAS. 2002. Statistical Analysis System, Versions 9.0 para Windows. Institute Inc., Cary, NC, USA. 
Stevenson, D. G.; Eller, F. J.; Wang, L.; Jane, J. L.; Wang, T. and Inglett, G. E. 2007. Oil and tocopherol content and composition of pumpkin seed oil in 12 cultivars. J. Agric. Food Chem. 55(10):4005-4013.

Terazawe, Y.; Masuda, R.; Yoshida, K. and Ito, K. 2001. Changes in carbohydrate composition in pumpkins (Kabocha) during fruit growth. ResearchGate. 70(5):656-658.

Vaštag, Ž.; Popović, L. J. and Popović, S. 2014. Bioactivity evaluation of cucurbitin derived enzymatic hydrolysates. IJABE. 8(5):445-448.

Young, K. M.; Jin, K. E.; Nam, K. Y.; Choi, C. and Hieu, L. B. 2012. Comparison of the chemical compositions and nutritive values of various pumpkin (Cucurbitaceae) species and parts. Nutr. Res. Practice. 6(1):21-27.

Ziaul, A. M.; Islam, T.; Rasel, U. M.; Jashim, U. M.; Mashiar, R. M. and Abdus, S. M. 2019. Comparative study on nutrient contents in the different parts of indigenous and hybrid varieties of pumpkin (Cucurbita maxima L.). Heliyon. 5(9):1-5. 